\title{
Tracking Quality of Low-Cost GNSS-Receivers
}

\author{
Johannes LIEBERMANN ${ }^{1}$, Gerhard NAVRATIL ${ }^{2}$ and Florian KRESSLER ${ }^{1}$ \\ ${ }^{1}$ AustriaTech, Vienna/Austria · johannes.liebermann@austriatech.at \\ ${ }^{2}$ Vienna University of Technology, Vienna/Austria
}

This contribution was double-blind reviewed as full paper.

\begin{abstract}
GNSS receivers are an essential part of modern technological devices. Mobile phones, digital cameras, and car navigational systems are only some examples for tools using GNSS technology. These sensors can be used to support other tasks as well. A simple example is fleet management where the car navigation systems can provide data on the current position, intended target and selected route of the vehicles. However, utilizing data requires information on its quality, in this case on the positional accuracy of the sensors. This quality was checked for GNSS receivers in different mobile phones and a tracking device.

Tracking of vehicles is usually restricted to a navigational network. In many cases, several lines may be in parallel with small lateral distance, e.g., lanes on a street, parallel streets, or parallel rail tracks. In these cases, the lateral quality is of higher importance than the longitudinal quality. Thus we concentrated on this aspect of the quality.
\end{abstract}

\section{Introduction}

Current technical devices often contain positioning sensors. Such a sensor then provides the location of the device and enables georeferencing data produced by the device. Examples for such devices are digital cameras or smartphones. Sometimes, the device itself is based on the existence of such a sensor, e.g., navigation systems. Usually the sensor is a chip evaluating signals received by an antenna. A standard sensor type for such purpose is a chip using signals from the American Global Positioning System (GPS) and other Global Navigation Satellite Systems (GNSS). One of the key properties of such solutions is that the sensor should not be too expensive. Thus low-cost sensors are typically used. Application development requires knowledge on the quality of the used data. If the quality level of the positioning is not sufficient, the application may not work. Expensive GNSS equipment and long observation times can procure relative positions with millimeter-precision. However, many applications, e.g., navigational support, have temporal constraints and thus long observation times are impossible. Thus a field test was performed to determine the tracking quality of GNSS sensors in currently used devices.

The remainder of the paper is organized as follows. In section 2 we explain the environment, in which the field test was performed. This discussion also contains a description of the tested devices and the test implementation. Section 3 contains the evaluation strategy and the results of the test. Final remarks conclude the paper. 


\section{Test Setup}

\subsection{Framework and detailed definition of the question}

The framework is defined by the research project "Testfeld Telematik" (telematics testing field, http://www.testfeld-telematik.at/home-en.html). The goal of the project is to develop and implement cooperative services for motorized individual traffic on Vienna's motorway triangle A4, A23 and S1 (figure 1). It should be evaluated, if the positing system GPS and low cost GPS receivers are capable nowadays of detecting lane changes.

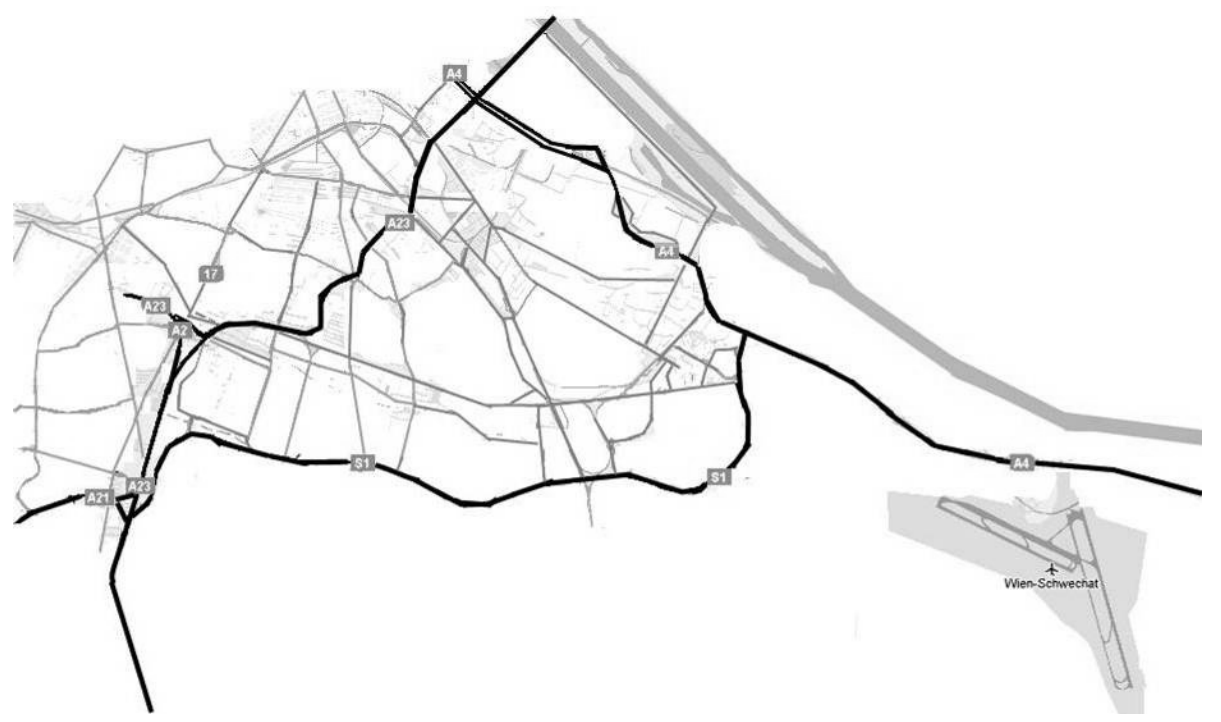

Fig. 1: Vienna's motorway triangle (source: Asfinag)

The key idea of the Testfeld Telematik is to provide accurate real-time traffic information through infrastructure to vehicle communication directly to the user and its in-car device. 50 test users equipped with aftermarket tablets, 10 cars with completely integrated solutions and 3000 ANDROID smartphone users will provide test data. There are two different approaches for the infrastructure to vehicle communication. One of them is the cellular approach, where GSM and UMTS are the preferred communication channels. The second approach foresees a direct communication between RSUs and an OBU. The overall goal of the Testfeld Telematik is to make traffic in Vienna safer, more sustainable, and more efficient.

In order to measure the effects of these cooperative services, an accompanying study called IMPAKT (Indikatoren zur multikriteriellen ParameterAnalyse kooperativer Transportsysteme, https://sites.google.com/a/impakt.at/impakt/) was created. In this study the effectiveness of the cooperative services in respect to traffic safety, traffic flow, environmental effects, and user acceptance are determined. The study is focused on detecting change in driver behaviour caused by traffic messages. This requires, among other pieces of informa- 
tion, GPS log-files from the test drivers. A first step is the detection of acceleration, deceleration, and lane changes. This is only possible, if the used devices can determine with sufficient accuracy. The achievable accuracy with low-cost receivers in post processing is the question discussed in this study.

The approach is to conduct a test run were GPS log files are being created from both, lowcost receivers and a highly accurate measuring unit. In post processing these files are analysed and the resulting positions compared. The important aspect for the project is the lateral offset. The highly accurate measuring unit consisted of:

- a JAVAD DELTA two frequency GNSS receiver with phase-calculation,

- $\quad$ an Inertial Navigation Unit with 3 gyroscopes, and

- a CORRSYS-DATRON Wheel Pulse Transducer.

The JAVAD DELTA receiver can use various signals including GPS L2C and L5, GLONASS C/A L2, and Galileo signals (JAVAD GNSS, 2012). The equipment was attached to a car as shown in figure 2 .
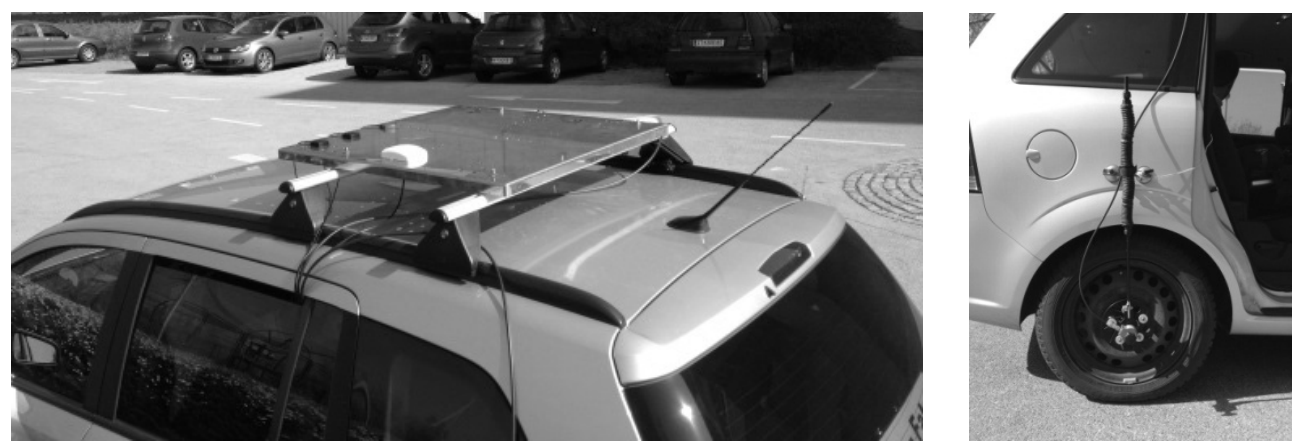

Fig. 2: Placement of the sensors: Demagnetized steel plate and porcelain antenna (left) for the GNSS observations and car-mounted CORRSYS (right) to track the wheel rotation.

The CORRSYS measures the travelled distance of the vehicle with a resolution of 2 millimetres, whereas the INU is recording the 6 degrees of freedom of cinematic motion at the same time. This includes acceleration and rotation of the vehicle. This method enables the creation of a reference trajectory even if there are no GPS signals available due to shadowing effects of obstacles (e.g., tunnels). During post processing, the Austrian Positioning Service (APOS) was used to improve the absolute position of the track. This led to an accuracy of about 2 centimetres for the trajectory.

In order to eliminate acceleration effects as far as possible, a constant speed of $80 \mathrm{~km} / \mathrm{h}$ was chosen. This is a compromise between possible ramp speeds and typical traffic speed. It caused the least amount of acceleration and deceleration. For large sections of the A4 and the A23, this is equal to the speed limit. 


\subsection{Tested low-cost receivers}

The following GNSS-receivers were tested:

- QSTARZ 1Hz GPS Tracker

- Apple IPhone 4

- HTC Desire

- $\quad$ Samsung Galaxy S II I9100

The QSTARZ tracker was used in the test because it will be used in IMPAKT to observe the behaviour of a representative cross section of drivers. The selected device, BTQ1000EX, is a GPS Lap Timer with $1 \mathrm{~Hz}$ logging of the position. It stores position (resulting in a track), speed, and distance. According to the producer, the Q1000EX is suitable for high paced sports like motor sports (QSTARZ, 2013). Table 1summarizes the most relevant information.

Table 1: BT-Q1000EX Product Specification

\begin{tabular}{|l|l|}
\hline General & MTK II GPS Module \\
\hline GPS Chip & L1, 1575.42MHz \\
\hline Frequency & $1.023 \mathrm{MHz}$ chip rate \\
\hline C/A Code & $66-$ CH Performance \\
\hline Channels & Built-in patch antenna with LNA \\
\hline Antenna (Internal) & Tracking $-165 \mathrm{dBm}$ \\
\hline Sensitivity & WGS84 \\
\hline Datum & Without aid: 3.0m 2D-RMS \\
\hline Performance Characteristic \\
\hline Position & $<3 \mathrm{~m}$ CEP(50\%) without SA (horizontal) \\
\hline Accuracy & DGPS (WAAS, ENGOS, MSAS): $2.5 \mathrm{~m}$ \\
\hline Velocity & Without aid: $0.1 \mathrm{~m} / \mathrm{s}$, DGPS (WAAS, ENGOS, MSAS): $0.05 \mathrm{~m} / \mathrm{s}$ \\
\hline Time & 50 ns RMS \\
\hline Cold/Warm/Hot Start & $35 / 33 / 1$ sec, average \\
\hline Dynamic Condition & $<18,000 \mathrm{~m}$ \\
\hline Altitude & $<515 \mathrm{~m} / \mathrm{sec}$ \\
\hline Velocity & $<4 \mathrm{~g}$ \\
\hline Acceleration & \\
\hline
\end{tabular}




\begin{tabular}{|l|l|}
\hline Protocol & $\begin{array}{l}\text { NMEA 0183 (V3.01) -GGA, GSA, GSV, RMC (Default) } \\
\text { VTG, GLL(Optional) }\end{array}$ \\
\hline Baud Rate & 115,200 bps \\
\hline Power & \\
\hline Built-in rechargeable Li-ion battery, Up to 42 hrs after fully charged \\
\hline Others & $72.2(\mathrm{~L}) \mathrm{X} 46.5(\mathrm{~W}) \mathrm{X} 20(\mathrm{H}) \mathrm{mm} / 64.7 \mathrm{~g}$ (battery included) \\
\hline Size / Weight & $-10^{\circ} \mathrm{C}$ to $+60^{\circ} \mathrm{C}$ \\
\hline $\begin{array}{l}\text { Operating } \\
\text { Temperature }\end{array}$ & $-20^{\circ} \mathrm{C}$ to $+60^{\circ} \mathrm{C}$ \\
\hline Storage Temperature
\end{tabular}

The different smartphones where used to compare it to other solutions provided by different hardware vendors. The measurements were done in April 2012 and thus the latest generation of smartphones (Apple iPhone 5 and Samsung Galaxy S III) is not yet included.

The test devices were placed behind the windscreen (compare figure 3 ) because this is a reasonable position for any user-added navigational device. This can cause shadowing problems by the car roof that could be avoided when designing built-in systems. On the other hand, the approach used in the test guarantees a realistic test scenario.

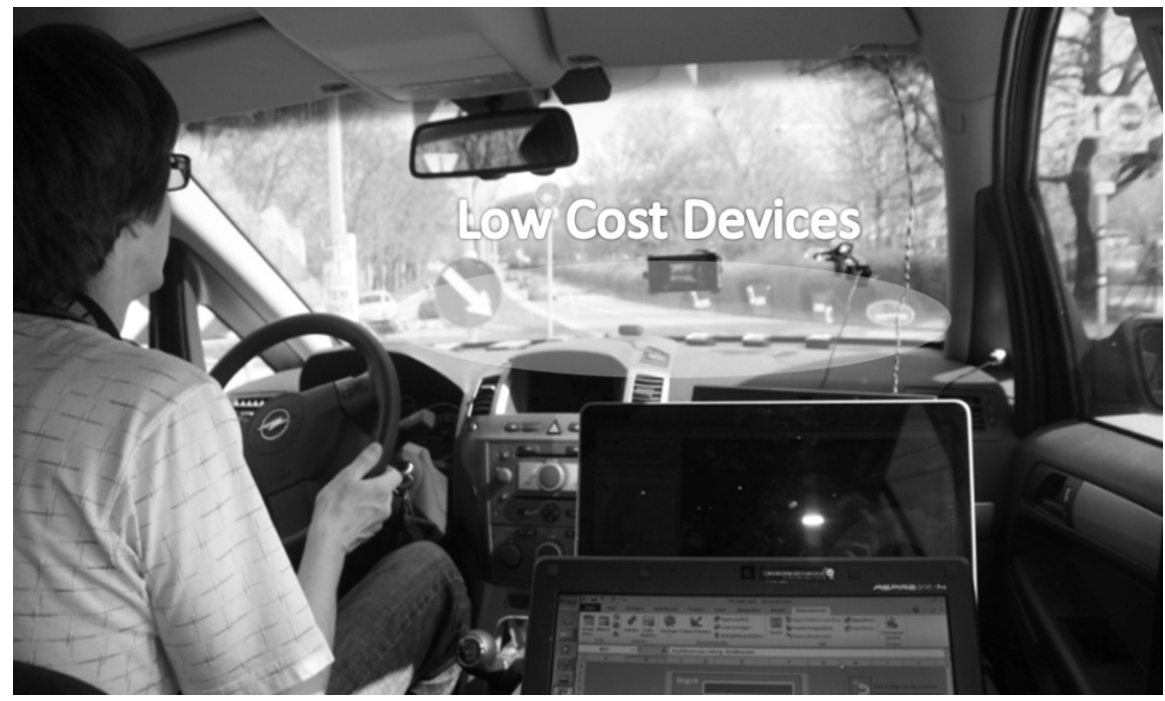

Fig. 3: Position of the low cost devices (picture: TraffiCon $\mathrm{GmbH}$ ) 


\section{Evaluation and Results}

The result of the test was logged points for all four test devices and a precise track from the highly accurate measurement unit. The track from the highly accurate measurement unit logger is shown in Figure 4. Start and end of the track are in the north-eastern part of the test area. The observation time was slightly over 30 minutes.

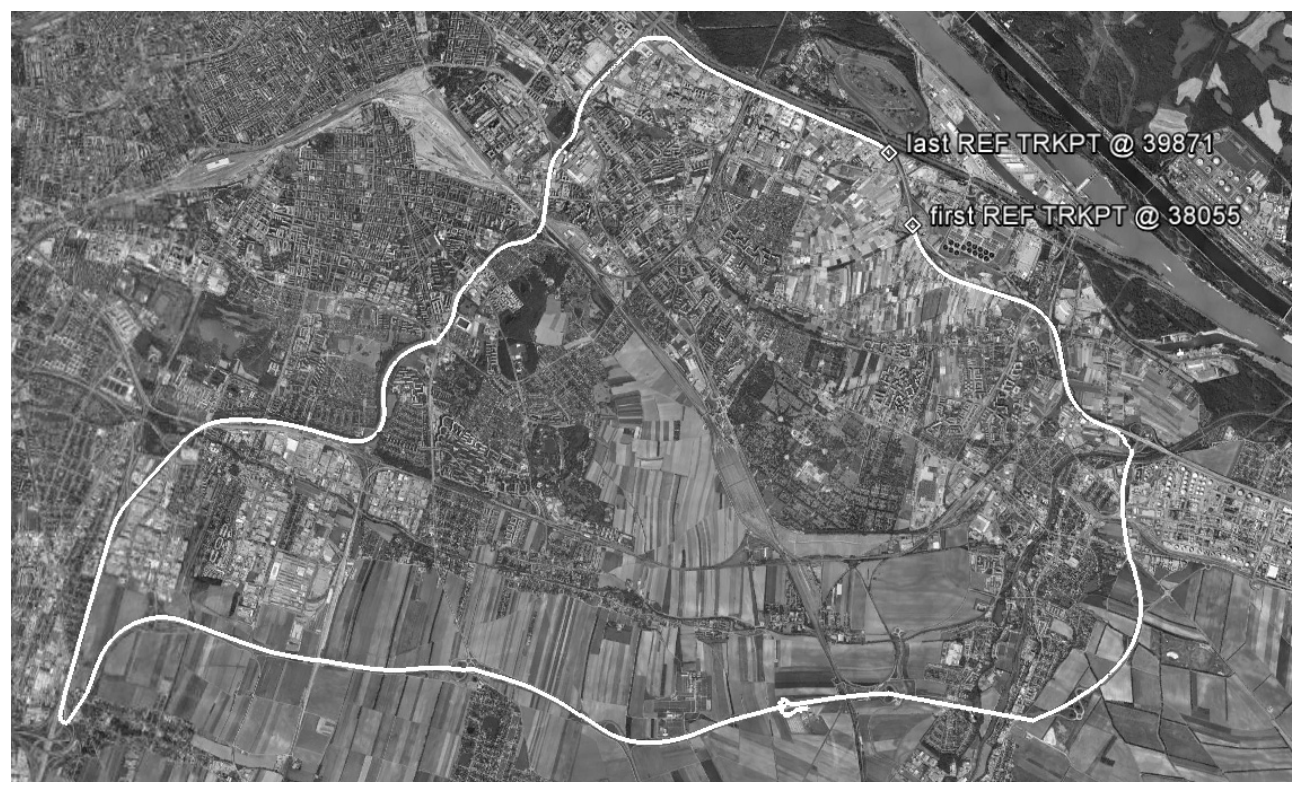

Fig. 4: Track created from logged coordinates

Figure 5 shows a first comparison of the reference log with the log from the QSTARZ logger. Mostly, the two logs are consistent. However, a few problematic areas can be identified. Firstly, in several places the QSTARZ logger could not store coordinates. The reason for this is the shadowing effect of tunnels and bridges in the test area. Secondly, a technical stop was necessary during the test run to recalibrate the CORRSYS-system as well as the INU. Figure 6 shows the detail from the southern part of the track where a long tunnel prevented the measurement of GPS positions and the effect of the recalibration stop on the observed location. The recalibration was necessary to eliminate measurement errors that result from an inevitable drift in the system. Recalibration requires a complete still stand of the vehicle. 


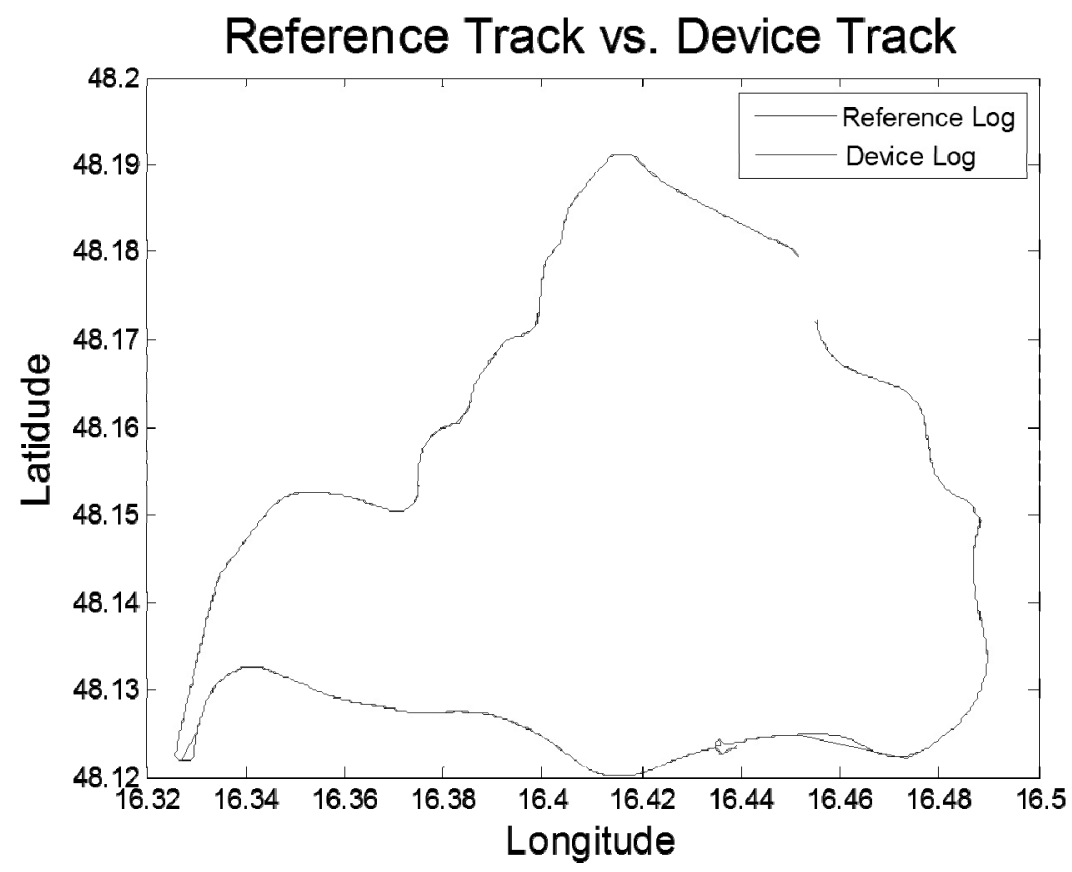

Fig. 5: $\quad$ Plot of QSTARZ-track (Device Log) and reference log

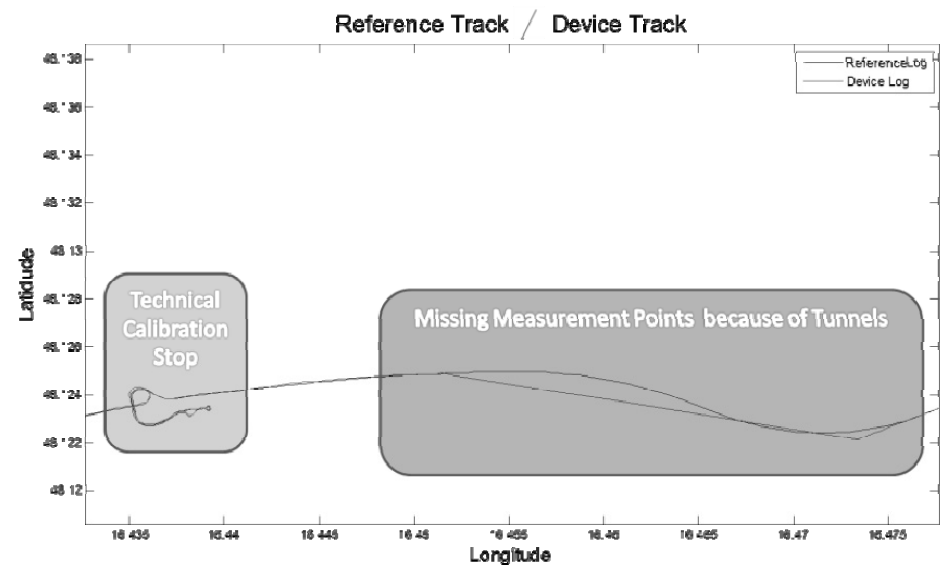

Fig. 6: Problem areas tunnel and technical stop

Estimation of measurement accuracy requires elimination of these erroneous points. Since the position of the tunnels was known, the positions could be used to eliminate all affected parts of the log files. Bridges did not influence the measurement significantly and observations near bridges were thus not eliminated. 
A problem for the analysis was finding the matching pairs. All positions do have time stamps. The GNSS positions have time stamps from GPS and are thus comparable. However, the accurate track has its time stamps from the INS. There is a small bias between these two time systems, which could not be compensated. The offset resulted in a longitudinal offset of roughly $20 \mathrm{~m}$. Due to measurement uncertainty of the low-cost receivers, the offset had a standard deviation of almost $5 \mathrm{~m}$. Since the curvature on highways is usually low, we chose not to compensate for the longitudinal offset because the effect on the lateral offset is minimal.
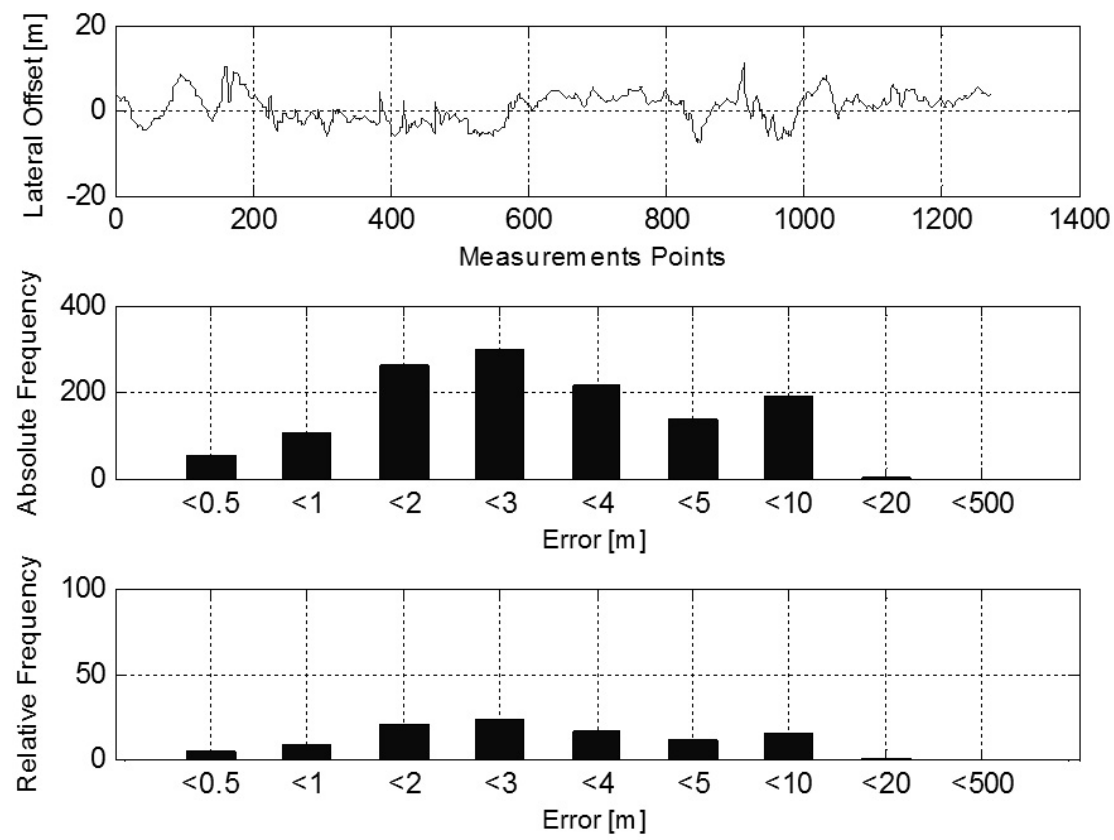

Fig. 7: $\quad$ Lateral Offset [m] for the QSTARZ GPS Logger

The average lateral offset is $0.7 \mathrm{~m}$ with a standard deviation of $3.5 \mathrm{~m}$. Thus $68 \%$ of the samples have a lateral offset between $-2.8 \mathrm{~m}$ and $+4.2 \mathrm{~m}$. Lane width on highways is less than $4 \mathrm{~m}$. Thus without additional improvement there will be a large overlap between computed positions of cars driving on different lanes.

The results for the other low-cost receivers were similar to the QSTARZ. Table 2 summarizes the results for the different receivers. 
Table 2: Comparison of the different results

\begin{tabular}{|l|l|l|}
\hline Device & Offset & Standard Deviation \\
\hline QSTARZ & $0.8 \mathrm{~m}$ & $3.5 \mathrm{~m}$ \\
\hline iPhone 4 & $-1.0 \mathrm{~m}$ & $3.0 \mathrm{~m}$ \\
\hline Desire & $1.3 \mathrm{~m}$ & $2.3 \mathrm{~m}$ \\
\hline Galaxy S II & $1.0 \mathrm{~m}$ & $2.4 \mathrm{~m}$ \\
\hline
\end{tabular}

A strange problem occurred with the QSTARZ GPS logger. It should be capable of using EGNOS signals to improve the positioning results. The option of using EGNOS was turned on during the test run but the device did not use the signals although EGNOS did not have a transmission problem during the test run as seen on http://egnos-usersupport.esspsas.eu/egnos_ops/data_gaps. A second test run to eliminate that problem failed due to clock problems. Further experiments were not possible due to time constraints of the used equipment.

\section{Conclusions}

The analysis of the performance, from the different devices, shows that the standard deviation of the lateral offset lies between 2.3 and 3.5 metres. Considering a lane width of 3.5 metres, a standard deviation of the same order is by far too inaccurate to detect a vehicles' lane change. It is interesting to note that the device with the sole purpose of tracking positions, the QSTARZ GPS logger has the highest standard deviation for the lateral offset. The HTC Desire produced the best results with a standard deviation of $2.3 \mathrm{~m}$. However, the offset of the HTC Desire is the largest in the group of devices.

IMPAKT discovered several problems in detecting vehicle lane changes with low cost GPS receivers as well. These problems occurred especially on curves. Therefore the project focuses the topic lane changes detection only on the motorway A4. For this section a street graph for each lane will be created to detect lane changes in post processing through a sophisticated map matching algorithm.

\section{References}

JAVAD GNSS (2012), JAVAD GNSS DELTA Operator's Manual (p. 54). San Jose, CA. http://javad.com/downloads/javadgnss/manuals/hardware/Delta_Operators_Manual.pdf.

QSTARZ (2013), Shop QSTARZ, Retrieved January 21, 2013, from

http://shopqstarz.com/. 
